\title{
Bandgap Control in Two-Dimensional Semiconductors via Coherent Doping of Plasmonic Hot Electrons
}

\section{Yu-Hui Chen}

Beijing Institute of Technology

\section{Ronnie Tamming}

Victoria University of Wellington

\section{Kai Chen}

Victoria University of Wellington

\section{Zhepeng Zhang}

Peking University

\section{Fengjiang Liu}

Westlake University https://orcid.org/0000-0002-0161-2290

\section{Yanfeng Zhang}

Peking University https://orcid.org/0000-0003-1319-3270

\section{Justin Hodgkiss}

Victoria University of Wellington

\section{Richard Blaikie}

The MacDiarmid Institute

\section{Boyang Ding ( $\sim$ boyang.ding@otago.ac.nz )}

University of Otago https://orcid.org/0000-0002-6299-5010

\section{Min Qiu}

Westlake University https://orcid.org/0000-0002-4613-5125

\section{Article}

Keywords: semiconductor technologies, optoelectronic devices, photonic devices

Posted Date: December 29th, 2020

DOI: https://doi.org/10.21203/rs.3.rs-124918/v1

License: (c) (1) This work is licensed under a Creative Commons Attribution 4.0 International License.

Read Full License 
Version of Record: A version of this preprint was published at Nature Communications on July 15th, 2021. See the published version at https://doi.org/10.1038/s41467-021-24667-8. 


\title{
Bandgap Control in Two-Dimensional Semiconductors via Coherent Doping of Plasmonic Hot Electrons
}

\author{
Teaser-Using strong plasmon-exciton coupling to flexibly modify bandgap in \\ two-dimensional semiconductors

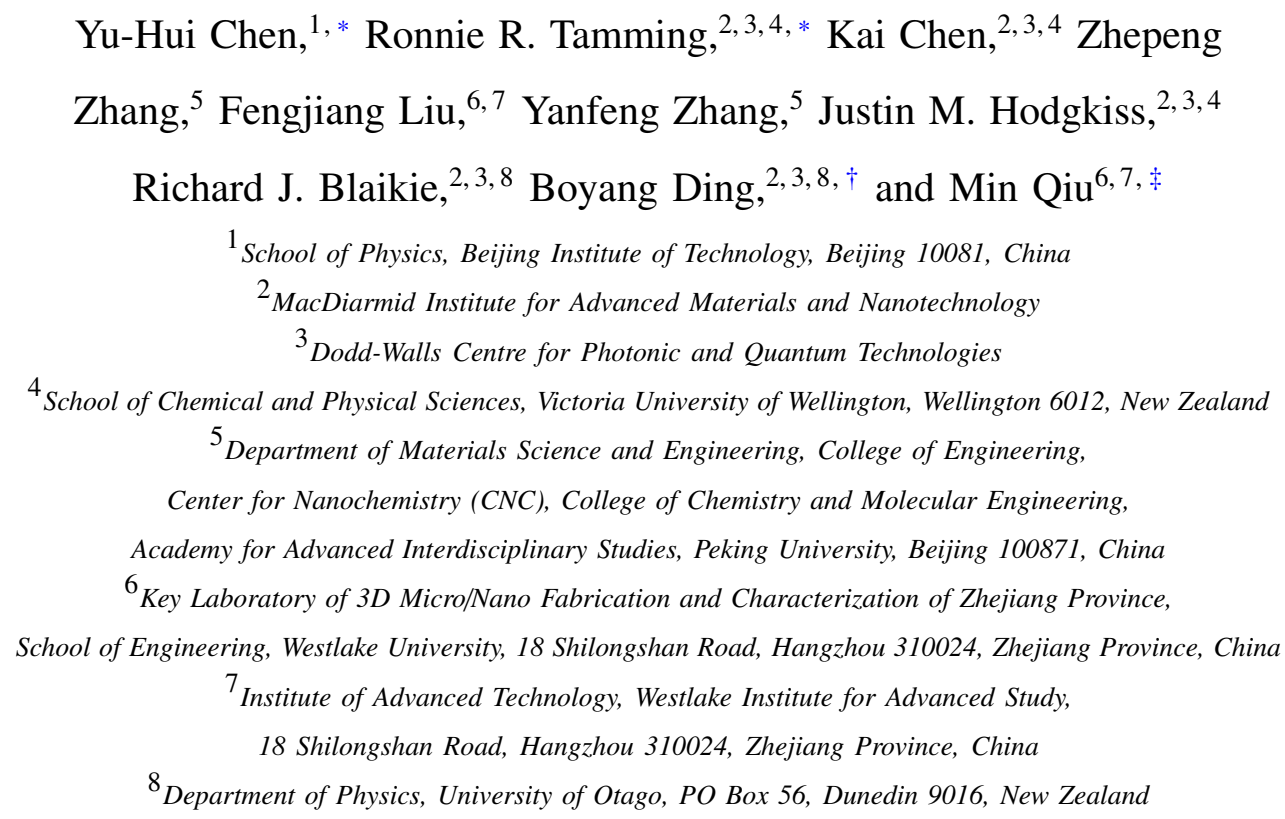

(Dated: December 9, 2020)

Bandgap control is of central importance for semiconductor technologies. The traditional means of control is to dope the lattice chemically, electrically or optically with charge carriers. Here, we demonstrate for the first time a widely tunable bandgap (renormalisation up to $650 \mathrm{meV}$ at room-temperature) in two-dimensional (2D) semiconductors by coherently doping the lattice with plasmonic hot electrons. In particular, we integrate tungsten-disulfide $\left(\mathrm{WS}_{2}\right)$ monolayers into a self-assembled plasmonic crystal, which enables coherent coupling between semiconductor excitons and plasmon resonances. Accompanying this process, the plasmon-induced hot electrons can repeatedly fill the $\mathrm{WS}_{2}$ conduction band, leading to population inversion and a significant reconstruction in band structures and exciton relaxations. Our findings provide an innovative and effective measure to engineer optical responses of 2D semiconductors, allowing a great flexibility in design and optimisation of photonic and optoelectronic devices. 


\section{Introduction}

Two-dimensional (2D) semiconductors, such as transition metal dichalcogenides (TMDCs)[1, 2], have direct bandgap at their monolayer limit, exhibiting tremendous potential in development of next-generation nanoscale devices. Like in their bulk counterparts, bandgap control plays a vital role in 2D semiconductor technoglogies, since it enables the creation of desirable optoelectronic properties that are required in numerous applications, ranging from lasers[3] to modulators[4], photodetectors[5] and photocatalysis[6]. The traditional means of control is to dope the lattice chemically[7], electrically [8] or optically [9] with charge carriers, the practicality of which is, however, limited by many factors, e.g. the irreversible bandgap modification, contact-type control and requirement of ultrastrong pump.

Here we report that one can effectively modify the electronic band structures of 2D semiconductors by establishing coherent strong coupling between the semiconductor excitons and a plasmonic resonator[10, 11]. In particular, plasmonic resonators are metallic nanostructures that support collective oscillation of electrons, known as plasmons. The excitation of plasmons can produce hot electrons, i.e. highly energetic electrons with non-equilibrium thermal distributions[12, 13], which, when plasmons are strongly coupled to 2D semiconductors, can repeatedly dope the lattice along with the coherent plasmon-exciton energy exchange. As a result, the bandgap of 2D semiconductors is significantly renormalised and the renormalisation can be easily altered via detuning plasmons from excitons.

\section{Results}

The schematic of our sample in Fig.1a demonstrates a $\mathrm{WS}_{2}$ monolayer (ML) deposited onto a plasmonic crystal (PC)[14, 15], which comprises of a periodic array of silver capped silica nanospheres that are coated with an ultrathin $\mathrm{Al}_{2} \mathrm{O}_{3}$ spacer with a thickness of $t=2.5 \pm 2 \mathrm{~nm}$. This metal-insulator-semiconductor configuration constitutes $\mathrm{PC}-\mathrm{WS}_{2}$ hybrid systems, supporting plasmon lattice modes propagating on the $\mathrm{PC}^{-\mathrm{WS}_{2}}$ interface. Here the top $\mathrm{WS}_{2} \mathrm{MLs}$ belong to the family of atomically thin TMDCs, having been extensively studied[16-20] for their unusual exciton-dominated optical responses, such as high absorption and emission efficiency. These properties make the $\mathrm{PC}-\mathrm{WS}_{2}$ systems a suitable platform to study plasmon-exciton interactions[15].

The PC geometries were chosen to excite plasmon lattice modes[10,11,14,15] that can match the frequency of exciton $\mathrm{A}(E=2.061 \mathrm{eV})$ in $\mathrm{WS}_{2} \mathrm{MLs}$ at certain incident angles $\theta$. Specifically, the plasmon modes show red-shift dispersion at higher $\theta$ (yellow curve in Fig.1b), which can be 

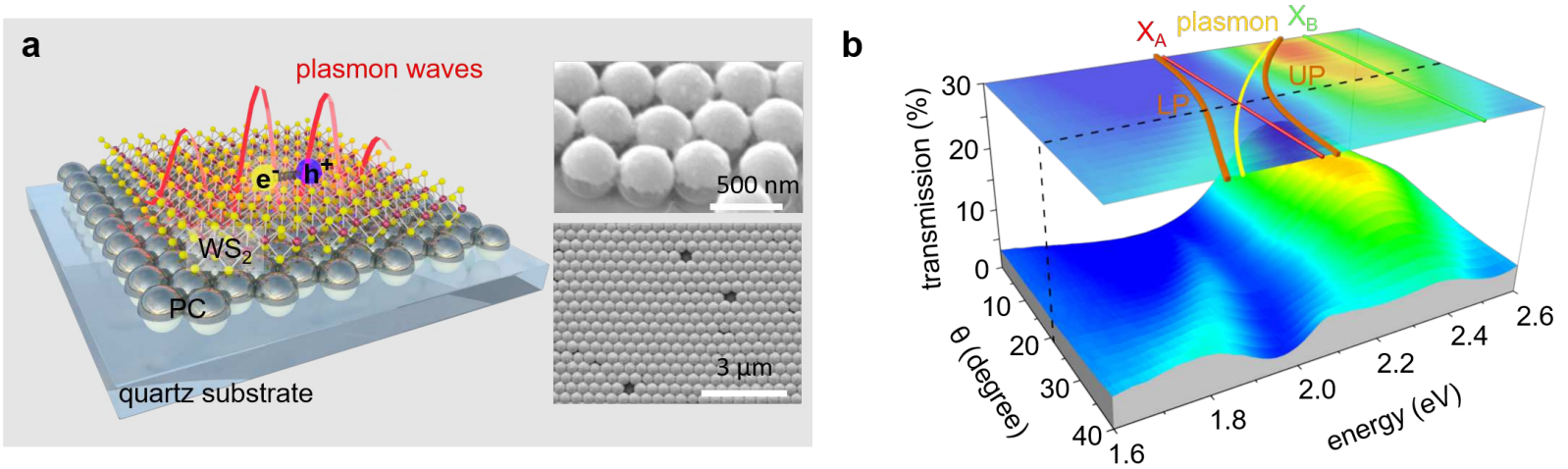

FIG. 1. Structures of a PC-WS $\mathbf{S}_{\mathbf{2}}$ sample and steady-state optical properties. a, schematic of polariton formation in a $\mathrm{WS}_{2} \mathrm{ML}$ that is supported on a self-assembled plasmonic crystal. $\mathrm{The} \mathrm{Al}_{2} \mathrm{O}_{3}$ spacer is not depicted for similicity. right insets: side and top-view scanning electron microscope (SEM) images; b, angle-resolved transmission spectra under p-polarised illumination and their projection (top $\mathrm{x}-\mathrm{y}$ plane), in which the spectral positions of exciton $\mathrm{A}\left(\mathrm{X}_{\mathrm{A}}\right)$ and $\mathrm{B}\left(\mathrm{X}_{\mathrm{B}}\right)$, calculated dispersions of plasmon lattice modes (yellow curve), and upper and lower branches of polaritons (orange curves) are indicated. The tuned angle $\left(\theta=22^{\circ}\right)$ is marked with a blacked dahsed line. Refer to Section 1 in the SI for detailed discussion of the strong plasmon-exciton coupling and its dispersion.

tuned in resonance with exciton $\mathrm{A}$ at $\theta=22^{\circ}$. As the result, plasmon modes coherently couple with excitons, leading to the formation of plasmon-exciton polaritons, i.e. half-light half-matter quasiparticles that inherit properties from both the plasmonic and excitonic components. Such strong coupling presents as splitting transmission maxima that follow the dispersions of upper polariton (UP) and lower polariton (LP), and the hybrid system is characterised by a vacuum Rabi splitting of $\hbar \cdot \Omega_{\mathrm{R}} \approx 136 \mathrm{meV}$. More detailed analysis of strong plasmon-exciton coupling in equilibrium states can be found in a previous work[15] and Fig.S1 in the Supplementary Information (SI).

Upon photoexcitation by an optical pulse $(3.1 \mathrm{eV}$ and $100 \mathrm{fs})$, the transient optical responses of PC-WS 2 samples can be characterised using femtosecond transient absorption (TA) spectroscopy (Fig.2a and Methods), which enables incident angle-resolved probes of the system's relaxation dynamics[21]. Fig.2b shows the tuned state $\left(\theta=22^{\circ}\right)$ transient transmission spectra $(\Delta \mathrm{T} / \mathrm{T})$ under a pump fluence of $12 \mu \mathrm{J} / \mathrm{cm}^{2}$, in which the polaritonic system displays two split relaxation traces that flank the spectral position of exciton A, apparently corresponding to UP and LP. This ultrashort timescale result confirms again the strong coupling nature of the $\mathrm{PC}-\mathrm{WS}_{2}$ systems.

When the lattice plasmons are detuned from exciton A, e.g. at $\theta=30^{\circ}$, the resonances redshift to $\sim 1.9 \mathrm{eV}$ (Fig.S1 in SI). In this case, the transient spectra (Fig.2c) only show a single relaxation trace at $2.07 \mathrm{eV}$, which, by comparing with $\Delta \mathrm{T} / \mathrm{T}$ spectra of bare $\mathrm{WS}_{2}$ MLs (Fig.S2 in SI), can be attributed to exciton A. However, no relaxation features from the detuned plasmons 
are observed. We note that the plasmon features are also absent in $\Delta \mathrm{T} / \mathrm{T}$ of bare PCs (Fig.S13 in SI). As a previous study[22] pointed out, this is induced by the off-resonance frequency (3.1 $\mathrm{eV}$ ) of the pump compared to the plasmon resonances, which results in very ineffective excitation of plasmons. These results indicate that the bare lattice plasmons can not be effectively excited without coupling to excitons under the pump conditions that can easily excite excitons.
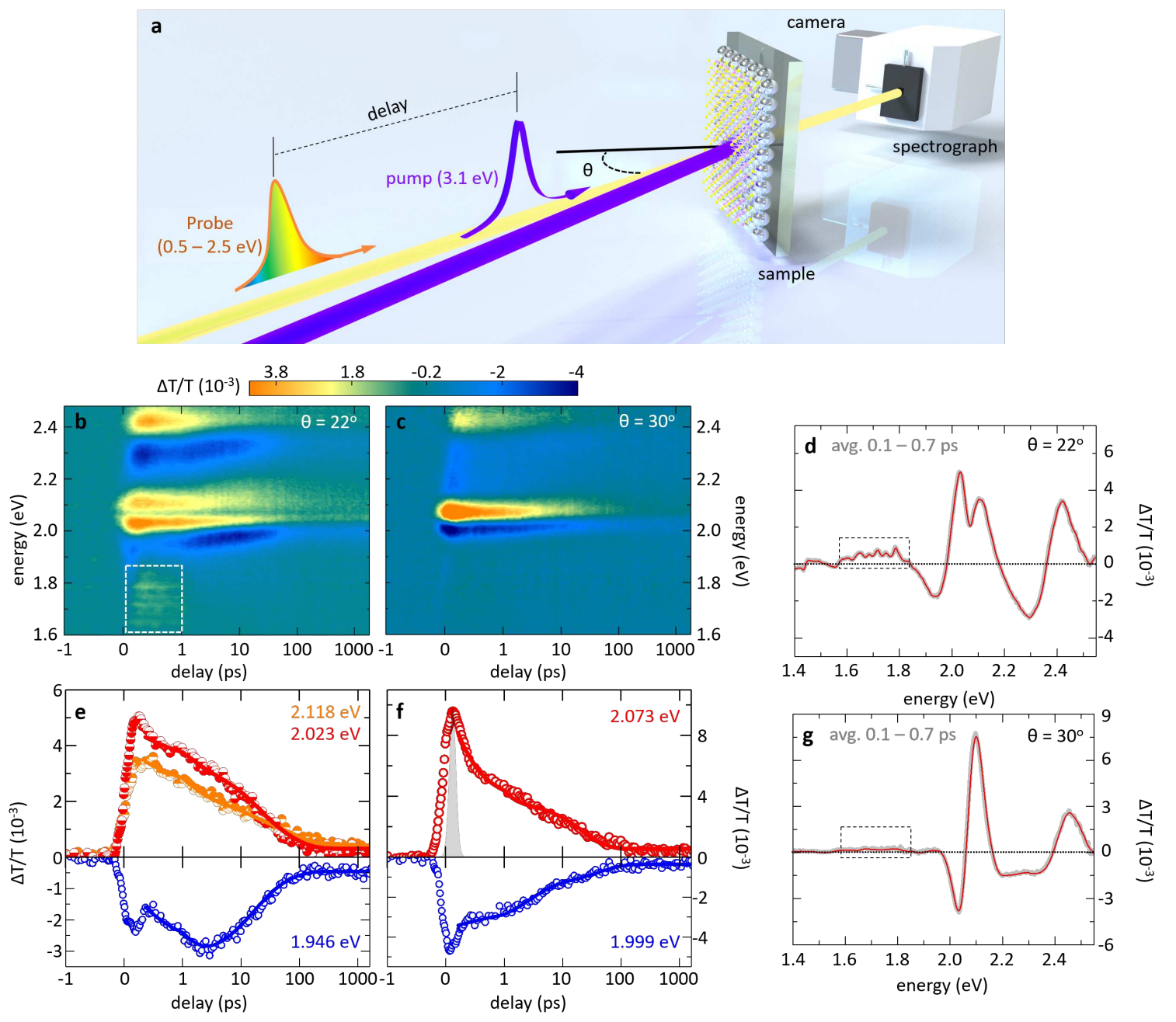

FIG. 2. Transient optical responses of PC-WS 2 systems. a, schematic of angle-resolved ultrafast pumpprobe spectroscopy; (note: the pump and probe beams are nearly colinear in practical experiments) b, $\mathbf{d}$ and $\mathbf{e}$ refer to normalised differential transmission spectra $(\Delta \mathrm{T} / \mathrm{T})$ at the tuned angle $\left(\theta=22^{\circ}\right)$, while $\mathbf{c}, \mathbf{f}$ and $\mathbf{g}$ refer to $\Delta \mathrm{T} / \mathrm{T}$ at the detuned angle $\left(\theta=30^{\circ}\right) ; \mathbf{b}$ and $\mathbf{c}$ are intensity plots of $\Delta \mathrm{T} / \mathrm{T}$ as function of time delay and probe photon energy, using the same colour bar (which is also used by Fig.3a); $\mathbf{d}$ and $\mathbf{g}$ are $\Delta \mathrm{T} / \mathrm{T}$ spectra averaged within the time span from 0.1 to 0.7 ps after pump; $\mathbf{e}$ and $\mathbf{f}$ are $\Delta \mathrm{T} / \mathrm{T}$ transient at specific energies (labelled with different colours), in which scatter symbols and solid curves represent measured and fitted data, respectively. Dashed frames in panel $\mathbf{b}, \mathbf{d}$ and $\mathbf{g}$ mark the spectral region of the broad maxima (see main text). All measurements were carried out using $400 \mathrm{~nm}(E=3.1 \mathrm{eV})$ pump pulses that have $100 \mathrm{fs}$ duration and pump fluence of $12 \mu \mathrm{J} / \mathrm{cm}^{2}$ at room temperature. The instrument-response-function is shown as the grey area in panelf. All time axes are displayed in logarithmic scales. 
What is interesting is that when the PC-WS 2 system is in tune (Fig.2b), there appears a $\Delta \mathrm{T} / \mathrm{T}$ maximum lasting for $\sim 1 \mathrm{ps}$ at the frequency range from $E=1.6$ to $1.8 \mathrm{eV}$, which, in contrast, is remarkably weaker in the detuned state (Fig.2c) and is completely absent in bare $\mathrm{WS}_{2} \mathrm{MLs}$ (Fig.S2 in SI). From the integrated $\Delta \mathrm{T} / \mathrm{T}$ spectrum near zero probe delay (Fig.2d), we can see that the broad maximum has positive magnitudes, indicating negative optical absorption or positive gain, which sharply contrasts with the flat spectral features in not only the detuned polaritons (Fig.2g) but also the bare PC samples (Fig.S13 in SI).
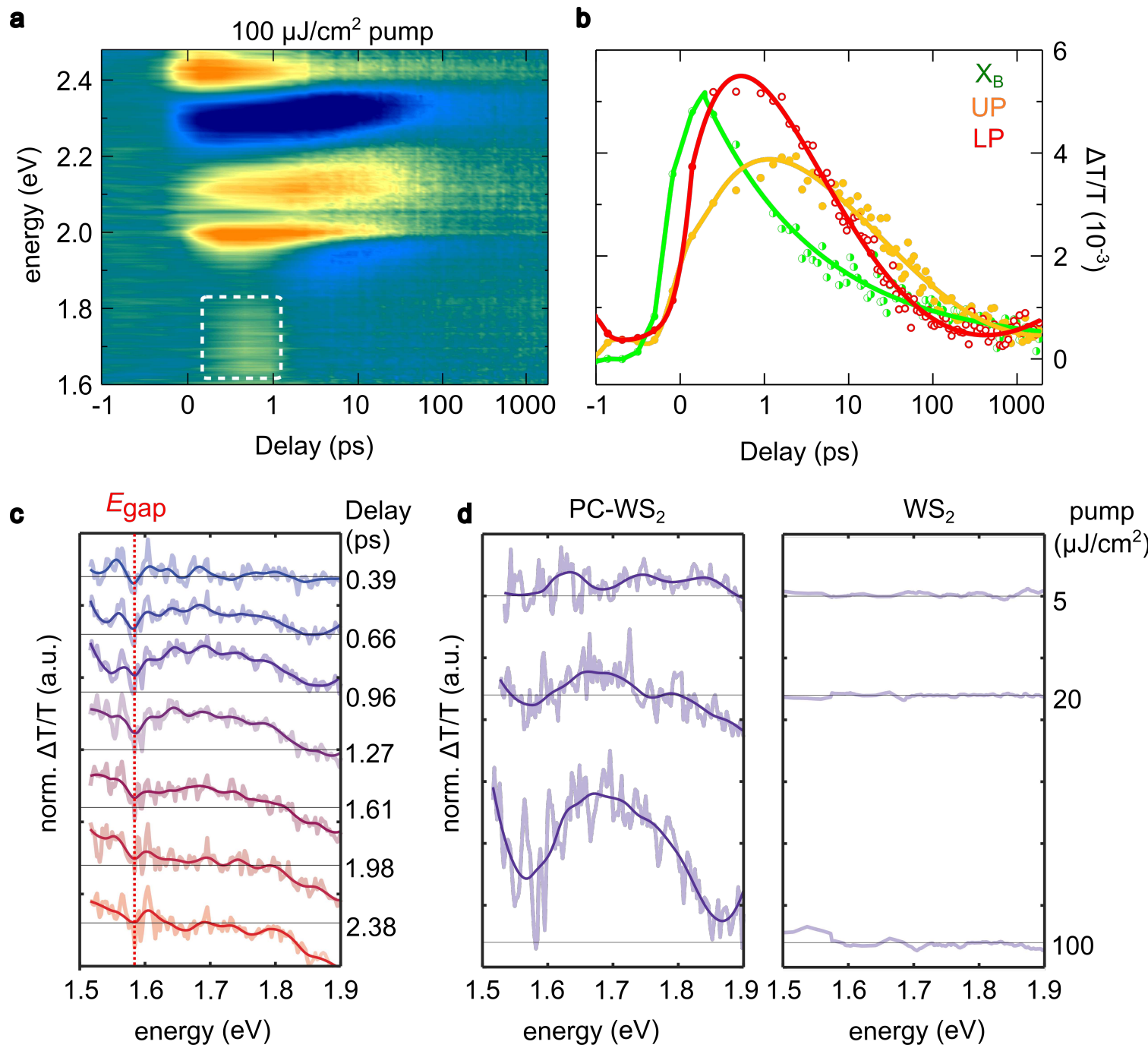

FIG. 3. Transient optical responses under high-power pump. a, intensity plot of $\Delta \mathrm{T} / \mathrm{T}$ spectra of PC$\mathrm{WS}_{2}$ under $100 \mu \mathrm{J} / \mathrm{cm}^{2}$ pump fluence at $\theta=22^{\circ}$, where orange (blue) colour represents the maximum (minimum) value. b, delay time dependent spectra $(\Delta \mathrm{T} / \mathrm{T})$ at energies of UP, LP and exciton B extracted from panel a. Solid curves are plotted only for visual guidance c, $\Delta \mathrm{T} / \mathrm{T}$ spectra at different delay times, extracted from the white dashed frame in panel $\mathbf{a}$; red dashed vertical line indicates the onset of renormalised bandgap. d, comparison of $\Delta \mathrm{T} / \mathrm{T}$ spectra at delay of 0.96 ps between PC-WS 2 (left) and $\mathrm{WS}_{2}$ MLs (right) under gradually increasing pump fluence. 
To confirm our observations, we have performed meaurements under $\sim 10$ times higher pump fluence $\left(100 \mu \mathrm{J} / \mathrm{cm}^{2}\right)$ for the tuned polaritons (Fig. 3a), where the broad maxima are not only present but much more pronounced than the low-power case. In addition, we can see large spectral shift (Fig.S9) as well as remarkably delayed occurance of UP and LP maxima (Fig.3b and Fig.S10), which indicate significant enhancement of the system's nonlinear optical responses (Details in Section 7 of SI). Similar to the low-power case, the transient variation of the broad maximum (Fig. 3c) takes $~ 1.5$ ps from initial excitation to fading. Fig. 3d shows the development of the broad maxima upon pump fluence, where the magnitude and width of the maximum are highly dependent on pump intensity. Under $100 \mu \mathrm{J} / \mathrm{cm}^{2}$ pump fluence, the full-width at halfmaximum reaches at $\sim 200 \mathrm{meV}$ with highly enhanced magnitudes as compared to the maximum under $5 \mu \mathrm{J} / \mathrm{cm}^{2}$ pump, also contrasting the flat spectral features in bare $\mathrm{WS}_{2}$ MLs.

\section{Discussions}

According to previous works $[9,23,24]$, the broad maxima are a clear evidence of bandgap renormalisation accompanied by population inversion, which is broadly understood as a population of high-density carriers in semiconductor lattice. In particular, when semiconductors are optically pumped, the generated non-equilibrium photo-carriers will occupy electron and/or hole states, leading to the formation of new quasiparticle bandgaps, which is described by[25]:

$$
\Delta E_{\mathrm{g}}=-\sum_{q \neq 0} V_{\mathrm{s}}(q)\left[f_{\mathrm{e}}(q)+f_{\mathrm{h}}(q)\right]-\sum_{q \neq 0}\left[V_{\mathrm{s}}(q)-V(q)\right]
$$

where $V_{\mathrm{s}}(q)$ and $V(q)$ represent fourier transforms of screened and unscreened Coulomb potentials, while $f_{\mathrm{e}}(q)$ and $f_{\mathrm{h}}(q)$ are occupation probabilities of electron and hole with momentum $q$. The onset of the new bandgap can be extracted from the low-energy end of the broad maximum. It means that in our experiments, the renormalised bandgap starts at $E_{\mathrm{g}} \approx 1.60 \mathrm{eV}$, lying $\sim 400 \mathrm{meV}$ below LP and $\sim 650 \mathrm{meV}$ below the initial bandgap of $\mathrm{WS}_{2}$ MLs (given that the binding energy of exciton $\mathrm{A}$ is $\sim 200 \mathrm{meV}[19])$.

These results, to the best of our knowledge, demonstrate the largest bandgap renormalisation in 2D semiconductors under such low pump intensities (e.g. down to $\sim 10 \mu \mathrm{J} / \mathrm{cm}^{2}$ ) to date. This sharply contrasts with similar observations[9] in bare $\mathrm{WS}_{2}$ single/bi-layers with much stronger photoexcitation $\left(840 \mu \mathrm{J} / \mathrm{cm}^{2}\right.$ at $70 \mathrm{~K}$ or $3400 \mu \mathrm{J} / \mathrm{cm}^{2}$ at room temperature). In their study, the ultrastrong pump enormously enhance exciton-exciton interactions, reducing exciton binding en- 
ergy, finally breaking excitons into unbound electron-hole plasma. This effect is known as Motttransition $[9,23]$, resulting in a high-density carrier population (up to $1 \times 10^{14} \mathrm{~cm}^{-2}$ ) in $\mathrm{WS}_{2}$ lattice. However, the pump power in our experiments is too low to develop a Mott-transition.

In addition, we note that the photoinduced absorption maxima (presenting as minima in negative $\Delta \mathrm{T} / \mathrm{T}$ magnitudes) in the tuned polaritons are clearly delayed as compared to its counterpart in the detuned polaritons (blue curves in Fig.2e and $2 \mathbf{f}$ and blue areas centred at $E=1.95$ $\mathrm{eV}$ in Fig.2b and 2c). In bare TMDC MLs[17-19, 26], such postponed absorption maxima are usually observed under high-intensity pumps (e.g. Fig.S2c and Fig.S3 in SI), which, similar to Mott-transition, owes to enhanced exciton-exciton and/or exciton-electron interactions induced by high-power pump (see Section $2 \& 6$ in SI for detailed discussions). In contrast, the delayed maxima in our hybrid systems appear under much weaker pump and are only associated with the tuned polaritons. Together with the bandgap renormalisation, all these evidences suggest the presence of additional carriers that highly relate to the strong coupling.

In a coupled system, resonant energy transfer (RET) may enhance carrier population. Specifically, plasmons can resonantly transfer absorbed optical energy to excitons through dipole-dipole interaction, which can facilitate the generation of photo-carriers in semiconductors[27] (See Section 3 in SI for details). However, as explained before [Fig.2c, 2g and Fig.S13 (SI)], the plasmon modes cannot be directly excited by the off-resonance pump but can only be excited by coupling to excitons. In this case, the carrier density in lattice cannot be enhanced by plasmonic RET due to energy conservasion.

We note that another source is more likely to contribute sufficient carriers, i.e. hot electrons from plasmonic crystals. Specifically, the excited plasmon modes may dephase from wavelike states through non-radiative decay, which generates electrons with non-equilibrium thermal distributions[13]. As a result, if plasmonic resonators are integrated with semiconductors, the excited electrons that have energies higher than the charge barrier formed at the metal-semiconductor interface can enter the lattice through direct electron transfer (DET)[28, 29], known as "hot electrons". As the barrier can prevent charges from returning back to the metals[27, 28], hot electrons can dope adjacent semiconductors[30], modifying their photovoltaic and photocatalytic performance[12]. (See Section 3 in SI for details of DET)

It is worth noting that, similar to the previous study[22], in our PC- $\mathrm{WS}_{2}$ systems the plasmonic hot electrons can only be generated through plasmon-exciton coupling. Specifically, after pumping, excitons in $\mathrm{WS}_{2}$ MLs are first excited and then coherently exchange energy with plasmons 


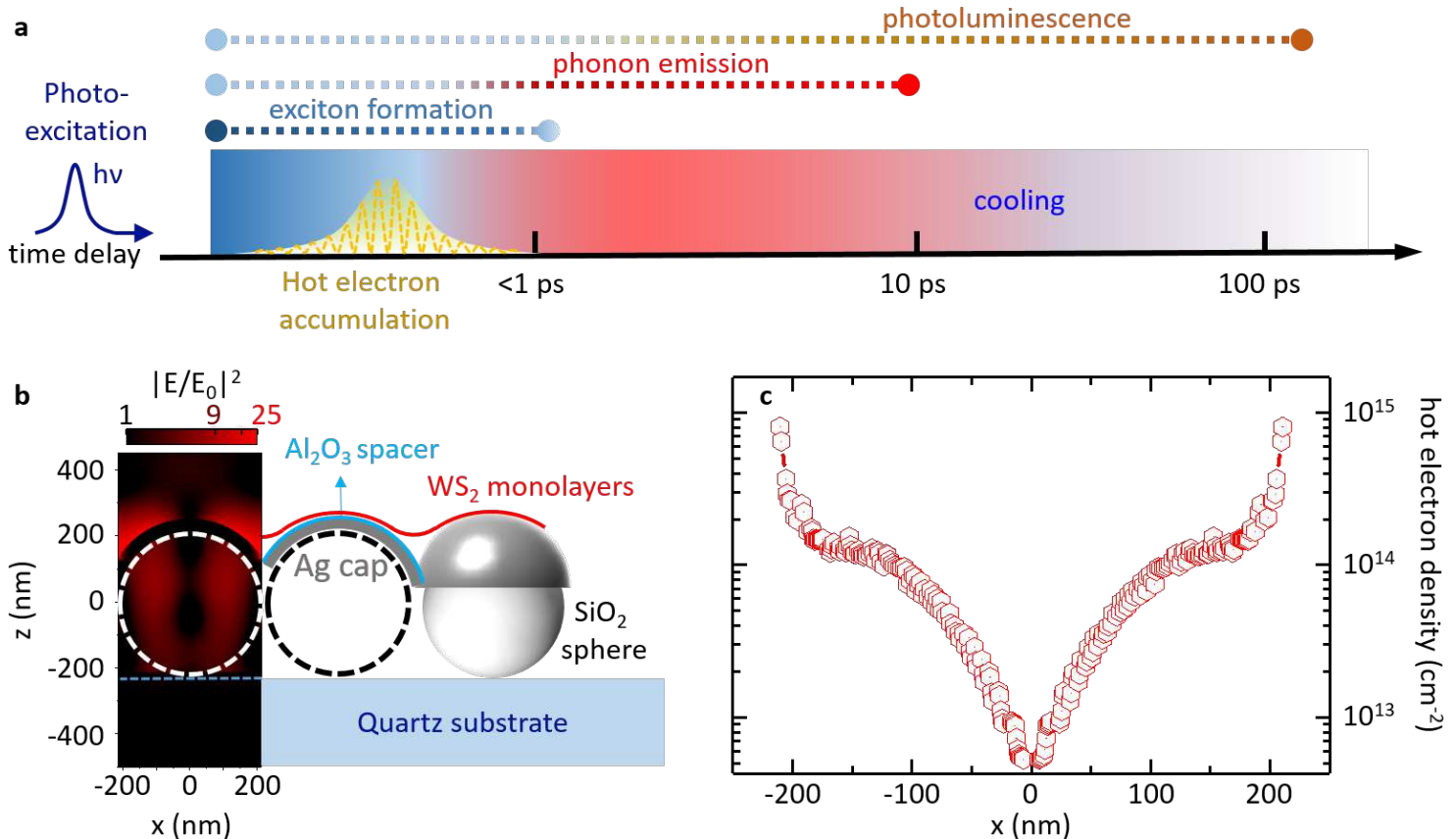

FIG. 4. Hot electron density and distribution. a, schematic illustration of the overall relaxation scenario after pulsed optical excitation on the PC-WS 2 systems, where the yellow oscillating dashed curve stands for repeated hot electron population in lattice; $\mathbf{b}$, simulated intensity enhancement $\left|\mathbf{E} / \mathbf{E}_{0}\right|^{2}$ in a single spherecap unit (XZ cross-section at middle of the sphere) at the frequency of $2.05 \mathrm{eV}$, together with a schematic of the PC-WS $\mathrm{W}_{2}$ sample; please note that the $\mathrm{WS}_{2} \mathrm{ML}$ is partially suspended at interstices between two Ag caps, but not fully covering the metal surface; detailed morphology can be referred to our previous work[15]; c, calculated hot electron density along the curved $\mathrm{WS}_{2}$ surface as a function of projected distance along $\mathrm{x}$-direction (at the same cross-section as in panel $\mathbf{b}$ ).

at the vacuum Rabi frequency $\left(\Omega_{\mathrm{R}} \approx 136 \mathrm{meV}\right)[31]$. As explained, the excitation of plasmons is accompanied by hot electron injection into the semiconductor. In other words, there is a fast and repeated hot electron population in the lattice that runs at an ultrashort period of $\sim 30 \mathrm{fs}$ $\left(T_{\mathrm{R}}=2 \pi / \Omega_{\mathrm{R}}\right)$.

As shown in the schematic (Fig.4a), such an ultrafast electron population is not only shorter than the non-radiative decay (at scales of $10 \mathrm{ps)}$ ) and the radiative decay process (up to few-hundred ps) in $\mathrm{WS}_{2} \operatorname{MLs}[17,18]$, but also greatly shorter than the exciton formation ( $\left.<1 \mathrm{ps}\right)[26]$. This means that during exciton relaxation, hot electrons can repeatedly fill the unoccupied states in conduction band of $\mathrm{WS}_{2}$ MLs. These injections can be evidenced by slower decays of both UP and LP (Fig.2e), compared with those of detuned states (Fig.2f and Table S2 in SI) and uncoupled excitons (Fig S2 and Table S1 in SI), because the electron population slows down the exciton bleaching via Pauli blocking and leads to extended lifetimes[22] (See Section 4 in SI for details). 
The generated hot electron density in our system can be calculated from electric fields[32]. The near-field enhancement $\left|\mathbf{E} / \mathbf{E}_{0}\right|^{2}$ in our structure has been modelled and shown in Fig.4b, where $\mathbf{E}_{0}$ refers to the incident field and $\mathbf{E}$ stands for the enhanced field by plasmonic excitations. The field distributes along the Ag cap surface with hot spots at the interstices between caps. Starting with the Hamiltonians of an electron Fermi gas, we have developed a model to calculate the hot electron number $N_{\mathrm{e}}$ per unit area at the $\mathrm{WS}_{2}$ surface (See Section 5 in SI for details):

$$
N_{\mathrm{e}}=\frac{1}{\pi} \frac{e^{2} E_{\mathrm{F}}^{2}}{\hbar} \frac{\hbar \omega-\Delta \phi_{\mathrm{TB}}}{(\hbar \omega)^{4}} \mid \mathbf{E}_{\mathrm{norm}} .^{2} \tau_{\text {pulse }}
$$

where $e$ is the electron charge, $\omega$ is the frequency, $E_{\mathrm{F}}$ is the $\mathrm{Ag}$ Fermi-level, $\mathbf{E}_{\text {norm. }}$ is the electric field normal to the surface and $\Delta \phi_{\mathrm{TB}}$ is set to be $1 \mathrm{eV}$ as the energy that is required to overcome the tunneling barrier formed at $\mathrm{Ag}-\mathrm{Al}_{2} \mathrm{O}_{3}-\mathrm{WS}_{2}$ interface[29, 33]. As shown in Fig.4c, upon illumination by a pulse that has $12 \mu \mathrm{J} / \mathrm{cm}^{2}$ fluence and $\tau_{\text {pulse }}=100 \mathrm{fs}$ duration (mimicking experimental conditions), along the $\mathrm{WS}_{2}$ surface, the density of electrons that are capable of overcoming the tunneling barrier is typically higher than $1 \times 10^{13} \mathrm{~cm}^{-2}$ with maxima values approaching $1 \times 10^{15}$ $\mathrm{cm}^{-2}$ at the cap interstices.

It is noted that some losses were not included in this calculation, e.g. energy dissipation during photoexcitation, exciton formation and exciton-to-plasmon transfer process. In addition, it is very hard to obtain the ratio of the generated hot electrons that enter $\mathrm{WS}_{2}$ lattice, because the current charge transfer models all build on phenomenological description, making the simulation tremendously complicated[34]. However, we note that the level of generated density is significantly higher than what is required to induce a large bandgap renormalisation. For example, the complete development of a Mott-transition in a $\mathrm{WS}_{2}$ monolayer only requires a carrier density up to $1 \times 10^{14} \mathrm{~cm}^{-2}$ [9]. In addition, a recent work[24] theoretically demonstrates that a charge doping density of $3.2 \times 10^{13} \mathrm{~cm}^{-2}$ in $\mathrm{WS}_{2}$ monolayers can already lead to a bandgap redshift up to 500 meV with carriers draining from conduction band $\mathrm{K}$ to $\Sigma$ valley[35] that renders the semiconductor indirect. These results share high similarity with our observation, suggesting that even if only a small portion (e.g. $1 / 100-1 / 10$ ) of the generated hot electrons dope the lattice, one can observe the large bandgap restructuring as in our experiments.

Given that there is little evidence for other possible carrier sources, e.g. polariton condensates [36], we conclude that coherent doping of plasmonic hot electrons is dominantly responsible for the spectral and transient features that require high-density population. In particular, the hot 
electron population starts with exciton formation and repeatedly takes place throughout the whole relaxation process. Owing to the existence of the tunneling barrier, hot electrons can be accumulated in the lattice before they decay (within 1 ps[13]), which simultaneously competes with rapid exciton relaxations, transiently converting the intrinsic $\mathrm{WS}_{2}$ monolayers to " $n$-doped" ones. This leads to the giant bandgap renormalisation with population inversion that peak at few-hundred femtoseconds (Fig.2b, Fig.3c and Fig.S11 in SI), and also induces the delayed maxima in Fig.2b and $2 \mathbf{e}$ (see Section 6 in SI for details). When we enhance pump power, the hot electron density is accordingly increased, even capable of delaying the occurance of excition bleaching (Fig.3b and Fig.S10 in SI).

\section{Summary}

As discussed above, the strong plasmon-exction coupling dramatically modifies the electronic band structures of $\mathrm{WS}_{2}$ monolayers, which are induced, to a large degree, by plasmonic hot electron doping via strong coupling. This effect is extremely hard to observe in traditional excitonpolaritons[36], being a non-trivial factor that has to be considered when studying light-matter interactions using plasmonic resonators, which, on the other hand, provides new and effective measures to engineer bandgap of 2D semiconductors. 


\section{METHODS}

1. sample preparation

Plasmonic crystals were prepared by self-assembly techniques together with thermal evaporation. In particular, silica spheres with diameter of $425 \mathrm{~nm}$ were self-assembled to a hexagonally packed monolayer on quartz substrates using a reported method[14], which is followed consecutively by evaporation of $40 \pm 5 \mathrm{~nm}$ thick silver film and $2.5 \pm 2 \mathrm{~nm}$ thick $\mathrm{Al}_{2} \mathrm{O}_{3}$ film. The metal evaporation acquires the shape of semi-spheres, resulting in an array of silver caps with a hexagonal lattice.

$\mathrm{WS}_{2}$ monolayers were prepared using chemical vapour evaporation with sulfur powder and tungsten dioxide powder being precursors, respectively. Using the poly(methyl methacrylate)assisted transfer method[37], we can transfer the prepared $\mathrm{WS}_{2}$ monolayer onto the PC surface. It is noted that the presence of $\mathrm{WS}_{2}$ monolayers is hardly seen from SEM images of our sample due to the ultrathin thickness of the monolayer $(<1 \mathrm{~nm})$. For more details about the fabrication and the monolayer morphology of the PC-WS 2 samples please see our previous publication[15].

\section{2. optical characterisation}

The large sample areas $\left(2-4 \mathrm{~cm}^{2}\right)$ allow us to conveniently perform femtosecond pump-probe absorption measurements[38] with different incident angles. Specifically, as shown in Fig.2a, the PC-WS 2 samples were excited by a pump pulse, followed by a delayed broadband illumination to probe the photoexcitation induced changes, which can be characterised by measuring the differential transmission, defined as the normalised change of the probe transmission induced by the pump $\Delta \mathrm{T} / \mathrm{T}=\left(\mathrm{T}-\mathrm{T}_{0}\right) / \mathrm{T}$, at different delayed times. The spectrometer in the pump-probe set-up is composed of a camera and a spectrograph, while the spectrograph is a home-built device using a prism to disperse the incident probe beam. The pump pulse has a frequency of $3.1 \mathrm{eV}(400 \mathrm{~nm})$, much higher than exciton A $(2.061 \mathrm{eV})$, while the probe pulse has a broadband frequency from 0.5 to $2.5 \mathrm{eV}$. The whole ultrafast system acquires a pulse resolution of $100 \mathrm{fs}$, and were converted to p-polarisation (electric field parallel to the plane of incidence) before entering samples. The transmission spectra of PC-WS 2 samples in equilibrium states (without photoexcitation) were preformed using the reported method[15]. All optical characterisations were carried out at room temperature. 


\section{Supplementary Information}

Section 1. Strong Coupling of Plasmons and Excitons

Section 2. Transient Optical Properties of $\mathrm{WS}_{2}$ monolayers

Section 3. Strong coupling induced charge transfer and generation

Section 4. Transient Analysis of Plasmon-Exciton Polaritons

Section 5. Theoritical Model of Hot Electrons in plasmonic crystal

Section 6. Delayed Photoinduced Absorption with Polaritons

Section 7. Nonlinear responses

Section 8. Additional Transient Absorption Spectra

\section{Acknowledgments}

Funding: The authors acknowledge the New Idea Research Funding 2018 (Dodd-Walls Centre for photonic and quantum technologies), the Marsden Fast-start Fund by Royal Society of New Zealand through contract MFP-UOO1827 and MFP-VUW1715 and the Smart Ideas Fund by Ministry of Business, Innovation and Employment, New Zealand through contract UOOX1802. In addition, this work was supported in part by the National Key Research and Development Program of China (no. 2017YFA0205700) and the National Natural Science Foundation of China (Nos. 6192782 and 51861135201), the Science and Technology Innovation Project of Beijing Institute of Technology. The authors also acknowledge the visiting Fellowship awarded by New Zealand Centre at Peking University. We thank Dr. M. Yan and Dr. F. Hong for their help with thin-film deposition, AFM, and SEM measurements. Author Contributions: B.D. and Y.-H.C. conceived the project; Z.Z, and B.D. prepared the samples; R.T., K.C., Y.-H.C., F.L. and B.D. carried out the optical and other characterization; Y.-H.C. and B.D. performed the simulation; Y.Z., M.Q., R.J.B., J.M.H. and B.D. supervised the projects; B.D. prepared the manuscript; all authors discussed and analyzed the results. Competing interests: The authors declare that they have no competing interests. Data and materials availability: All data needed to evaluate the conclusions in the paper are present in the paper and/or the Supplementary Materials. Additional data related to this paper may be requested from the authors. 


\section{REFERENCES}

* These authors contributed equally

† boyang.ding@otago.ac.nz

\#qiu_lab@westlake.edu.cn

[1] Kin Fai Mak, Changgu Lee, James Hone, Jie Shan, and Tony F. Heinz. Atomically thin MoS2: A new direct-gap semiconductor. Phys. Rev. Lett., 105(13):136805, 2010.

[2] Andrea Splendiani, Liang Sun, Yuanbo Zhang, Tianshu Li, Jonghwan Kim, Chi Yung Chim, Giulia Galli, and Feng Wang. Emerging photoluminescence in monolayer MoS2. Nano Lett., 10(4):1271$1275,2010$.

[3] Yu Ye, Zi Jing Wong, Xiufang Lu, Xingjie Ni, Hanyu Zhu, Xianhui Chen, Yuan Wang, and Xiang Zhang. Monolayer excitonic laser. Nat. Photonics, 9(October):733-737, 2015.

[4] Kin Fai Mak and Jie Shan. Photonics and optoelectronics of 2D semiconductor transition metal dichalcogenides. Nat. Photonics, 10(4):216-226, 2016.

[5] Oriol Lopez-Sanchez, Dominik Lembke, Metin Kayci, Aleksandra Radenovic, and Andras Kis. Ultrasensitive photodetectors based on monolayer MoS 2. Nat. Nanotechnol., 8(7):497-501, 2013.

[6] Damien Voiry, Hisato Yamaguchi, Junwen Li, Rafael Silva, Diego C.B. Alves, Takeshi Fujita, Mingwei Chen, Tewodros Asefa, Vivek B. Shenoy, Goki Eda, and Manish Chhowalla. Enhanced catalytic activity in strained chemically exfoliated WS2nanosheets for hydrogen evolution. Nat. Mater., 12(9):850-855, 2013.

[7] Jimin Kim, Seung Su Baik, Sae Hee Ryu, Yeongsup Sohn, Soohyung Park, Byeong-Gyu Park, Jonathan Denlinger, Yeonjin Yi, Hyoung Joon Choi, and Keun Su Kim. Observation of Tunable Band Gap and Anisotropic Dirac Semimetal State in Black Phosphorus. Science (80-. )., 349(6249):723$726,2015$.

[8] Alexey Chernikov, Arend M. Van Der Zande, Heather M. Hill, Albert F. Rigosi, Ajanth Velauthapillai, James Hone, and Tony F. Heinz. Electrical Tuning of Exciton Binding Energies in Monolayer WS2. Phys. Rev. Lett., 115(12):1-6, 2015.

[9] Alexey Chernikov, Claudia Ruppert, Heather M. Hill, Albert F. Rigosi, and Tony F. Heinz. Population inversion and giant bandgap renormalization in atomically thin WS2 layers. Nat. Photonics, 9(7):466- 
470, 2015.

[10] T. W. Ebbesen, H. J. Lezec, H. F. Ghaemi, T Thio, and P. A. Wolf. Extraordinary optical transmission through sub-wavelength hole arrays. Nature, 391(12):667-669, 1998.

[11] Haitao Liu and Philippe Lalanne. Microscopic theory of the extraordinary optical transmission. $\mathrm{Na}$ ture, 452(7188):728-731, 2008.

[12] César Clavero. Plasmon-induced hot-electron generation at nanoparticle/metal-oxide interfaces for photovoltaic and photocatalytic devices. Nat. Photonics, 8(2):95-103, 2014.

[13] Mark L Brongersma, Naomi J Halas, and Peter Nordlander. Plasmon-induced hot carrier science and technology. Nat. Nanotechnol., 10(1):25-34, 2015.

[14] Boyang Ding, Calin Hrelescu, Nikita Arnold, Goran Isic, and Thomas A. Klar. Spectral and Directional Reshaping of Fluorescence in Large Area Self-Assembled Plasmonic-Photonic Crystals. Nano Lett., 13:378-386, feb 2013.

[15] Boyang Ding, Zhepeng Zhang, Yu-Hui Chen, Yanfeng Zhang, Richard J Blaikie, and Min Qiu. Tunable Valley Polarized Plasmon-Exciton Polaritons in Two-Dimensional Semiconductors. ACS Nano, 13(2):1333-1341, 2019.

[16] Ziliang Ye, Ting Cao, Kevin O’Brien, Hanyu Zhu, Xiaobo Yin, Yuan Wang, Steven G. Louie, and Xiang Zhang. Probing excitonic dark states in single-layer tungsten disulphide. Nature, 513(7517):214$218,2014$.

[17] E. J. Sie, A. Steinhoff, C. Gies, C. H. Lui, Q. Ma, M. Roesner, G. Schoenhoff, F. Jahnke, T. O. Wehling, Y.-H. Lee, J. Kong, P. Jarillo-Herrero, and N. Gedik. Observation of Exciton RedshifBlueshift Crossover in Monolayer WS 2. Nano Lett., 17:4210-4216, 2017.

[18] Claudia Ruppert, Alexey Chernikov, Heather M. Hill, Albert F. Rigosi, and Tony F. Heinz. The Role of Electronic and Phononic Excitation in the Optical Response of Monolayer WS2 after Ultrafast Excitation. Nano Lett., 17(2):644-651, 2017.

[19] Paul D. Cunningham, Aubrey T. Hanbicki, Kathleen M. McCreary, and Berend T. Jonker. Photoinduced Bandgap Renormalization and Exciton Binding Energy Reduction in WS2. ACS Nano, 11(12):12601-12608, 2017.

[20] Alexander Steinhoff, Matthias Florian, Malte Rösner, Gunnar Schönhoff, Tim Oliver Wehling, and Frank Jahnke. Exciton fission in monolayer transition metal dichalcogenide semiconductors. Nat. Commun., 8:1166, 2017.

[21] Brendan L. Darby, Baptiste Auguié, Matthias Meyer, Andres E. Pantoja, and Eric C. Le Ru. Modified 
optical absorption of molecules on metallic nanoparticles at sub-monolayer coverage. Nat. Photonics, 10:40-45, 2016.

[22] Abdelaziz Boulesbaa, Viktoriia E. Babicheva, Kai Wang, Ivan I. Kravchenko, Ming Wei Lin, Masoud Mahjouri-Samani, Christopher B. Jacobs, Alexander A. Puretzky, Kai Xiao, Ilia Ivanov, Christopher M. Rouleau, and David B. Geohegan. Ultrafast Dynamics of Metal Plasmons Induced by 2D Semiconductor Excitons in Hybrid Nanostructure Arrays. ACS Photonics, 3(12):2389-2395, 2016.

[23] L. Meckbach, T. Stroucken, and S. W. Koch. Giant excitation induced bandgap renormalization in TMDC monolayers. Appl. Phys. Lett., 112(6), 2018.

[24] D. Erben, A. Steinhoff, C. Gies, G. Schönhoff, T. O. Wehling, and F. Jahnke. Excitation-induced transition to indirect band gaps in atomically thin transition-metal dichalcogenide semiconductors. Phys. Rev. B, 98(3):1-13, 2018.

[25] Nasser Peyghambarian, Stephan W. Koch, and Andre Mysyrowicz. Introduction to semiconductor optics. Prentice-Hall, Inc., Upper Saddle River, NJ, USA, 1993.

[26] F Ceballos, Q N Cui, M Z Bellus, and H Zhao. Exciton formation in monolayer transition metal dichalcogenides. Nanoscale, 8(22):11681-11688, 2016.

[27] Scott K. Cushing, Jiangtian Li, Fanke Meng, Tess R. Senty, Savan Suri, Mingjia Zhi, Ming Li, Alan D. Bristow, and Nianqiang Wu. Photocatalytic activity enhanced by plasmonic resonant energy transfer from metal to semiconductor. J. Am. Chem. Soc., 134(36):15033-15041, 2012.

[28] F. Pelayo García De Arquer, Agustín Mihi, Dominik Kufer, and Gerasimos Konstantatos. Photoelectric energy conversion of plasmon-generated hot carriers in metal-insulator-semiconductor structures. ACS Nano, 7(4):3581-3588, 2013.

[29] Xiang Tian Kong, Zhiming Wang, and Alexander O. Govorov. Plasmonic Nanostars with Hot Spots for Efficient Generation of Hot Electrons under Solar Illumination. Adv. Opt. Mater., 5(15):1-10, 2017.

[30] Zheyu Fang, Yumin Wang, Zheng Liu, Andrea Schlather, Pulickel M. Ajayan, Frank H.L. Koppens, Peter Nordlander, and Naomi J. Halas. Plasmon-induced doping of graphene. ACS Nano, 6(11):10222-10228, 2012.

[31] Parinda Vasa, Wei Wang, Robert Pomraenke, Melanie Lammers, Margherita Maiuri, Cristian Manzoni, Giulio Cerullo, and Christoph Lienau. Real-time observation of ultrafast Rabi oscillations between excitons and plasmons in metal nanostructures with J-aggregates. Nat. Photon., 7:128-132, 2013. 
[32] Hayk Harutyunyan, Alex B.F. Martinson, Daniel Rosenmann, Larousse Khosravi Khorashad, Lucas V. Besteiro, Alexander O. Govorov, and Gary P. Wiederrecht. Anomalous ultrafast dynamics of hot plasmonic electrons in nanostructures with hot spots. Nat. Nanotechnol., 10(9):770-774, 2015.

[33] Shan Zheng, Haichang Lu, Huan Liu, Dameng Liu, and John Robertson. Insertion of an ultrathin A12O3 interfacial layer for Schottky barrier height reduction in WS2 field-effect transistors. Nanoscale, 11(11):4811-4821, 2019.

[34] Garth A. Jones and David S. Bradshaw. Resonance Energy Transfer: From Fundamental Theory to Recent Applications. Front. Phys., 7:100, 2019.

[35] F. Lohof, A. Steinhoff, M. Florian, M. Lorke, D. Erben, F. Jahnke, and C. Gies. Prospects and Limitations of Transition Metal Dichalcogenide Laser Gain Materials. Nano Lett., 19(1):210-217, 2019.

[36] Tim Byrnes, Na Young Kim, and Yoshihisa Yamamoto. Exciton-polariton condensates. Nat. Phys., 10(11):803-813, 2014.

[37] Qingqing Ji, Yu Zheng, Yanfeng Zhang, and Zhongfan Liu. Chemical vapour deposition of group-VIB metal dichalcogenide monolayers: Engineered substrates from amorphous to single crystalline. Chem. Soc. Rev., 44(9):2587-2602, 2015.

[38] Joseph K. Gallaher, Shyamal K.K. Prasad, Mohammad A. Uddin, Taehyo Kim, Jin Young Kim, Han Young Woo, and Justin M. Hodgkiss. Spectroscopically tracking charge separation in polymer : fullerene blends with a three-phase morphology. Energy Environ. Sci., 8(9):2713-2724, 2015.

[39] Hui Deng, Hartmut Haug, and Yoshihisa Yamamoto. Exciton-polariton Bose-Einstein condensation. Rev. Mod. Phys., 82(2):1489-1537, 2010.

[40] Hangyong Shan, Ying Yu, Xingli Wang, Yang Luo, Shuai Zu, Bowen Du, Tianyang Han, Bowen Li, Yu Li, Jiarui Wu, Feng Lin, Kebin Shi, Beng Kang Tay, Zheng Liu, Xing Zhu, and Zheyu Fang. Direct observation of ultrafast plasmonic hot electron transfer in the strong coupling regime. Light Sci. Appl., 8(1), 2019.

[41] Tian Jiang, Runze Chen, Xin Zheng, Zhongjie Xu, and Yuhua Tang. Photo-induced excitonic structure renormalization and broadband absorption in monolayer tungsten disulphide. Opt. Express, 26(2):859, 2018.

[42] Frank Ceballos and Hui Zhao. Ultrafast Laser Spectroscopy of Two-Dimensional Materials Beyond Graphene. Adv. Funct. Mater., 27:1604509, 2016.

[43] Haining Wang, Changjian Zhang, and Farhan Rana. Ultrafast dynamics of defect-assisted electronhole recombination in monolayer MoS2. Nano Lett., 15(1):339-345, 2015. 
[44] Martin Amani, Der-Hsien Lien, Daisuke Kiriya, Rafik Addou, Angelica Azcatl, Jiyoung Noh, Surabhi R Madhvapathy, Rafik Addou, Santosh Kc, Madan Dubey, Kyeongjae Cho, Robert M Wallace, Si-chen Lee, Jr-hau He, Joel W Ager Iii, Xiang Zhang, Eli Yablonovitch, and Ali Javey. Nearunity photoluminescence quantum yield in MoS2. Science, 350(6264):1065-1068, 2015.

[45] Akihiro Furube and Shuichi Hashimoto. Insight into plasmonic hot-electron transfer and plasmon molecular drive: new dimensions in energy conversion and nanofabrication. NPG Asia Mater., 9(12):e454, 2017.

[46] XingXing Chen, Yu-Hui Chen, Jian Qin, Ding Zhao, Boyang Ding, Richard J. Blaikie, Min Qiu. Mode Modification of Plasmonic Gap Resonances induced by Strong Coupling with Molecular Excitons. Nano Lett., 17:3246-3251, 2017.

[47] Grant Aivazian, Hongyi Yu, Sanfeng Wu, Jiaqiang Yan, David G. Mandrus, David Cobden, Wang Yao, and Xiaodong Xu. Many-body effects in nonlinear optical responses of 2D layered semiconductors. 2D Mater., 4(2):025024, 2017.

[48] Marie Elena Kleemann, Rohit Chikkaraddy, Evgeny M. Alexeev, Dean Kos, Cloudy Carnegie, Will Deacon, Alex Casalis De Pury, Christoph Große, Bart De Nijs, Jan Mertens, Alexander I. Tartakovskii, and Jeremy J. Baumberg. Strong-coupling of WSe2 in ultra-compact plasmonic nanocavities at room temperature. Nat. Commun., 8(1):1296, 2018.

[49] V. Shahnazaryan, I. Iorsh, I. A. Shelykh, and O. Kyriienko. Exciton-exciton interaction in transitionmetal dichalcogenide monolayers. Phys. Rev. B, 96(11):115409, 2017.

[50] P Vasa, R Pomraenke, G Cirmi, and E De Re. Ultrafast Manipulation of Strong Coupling in MetalMolecular Aggregate Hybrid Nanostructures. ACS Nano, 4(12):7559-7565, 2010.

[51] Fábio Barachati, Antonio Fieramosca, Soroush Hafezian, Jie Gu, Biswanath Chakraborty, Dario Ballarini, Ludvik Martinu, Vinod Menon, Daniele Sanvitto, and Stéphane Kéna-Cohen. Interacting polariton fluids in a monolayer of tungsten disulfide. Nat. Nanotechnol., 13(10):906-909, 2018.

[52] Jian Qin, Zhepeng Zhang, Yu-Hui Chen, Yanfeng Zhang, Richard Blaikie, Boyang Ding, and Min Qiu. Revealing Strong Plasmon-Exciton Coupling Between Nano-gap Resonators and Two-Dimensional Semiconductors at Ambient Conditions. Phys. Rev. Lett., 124(6):63902, 2020. 


\section{Figures}

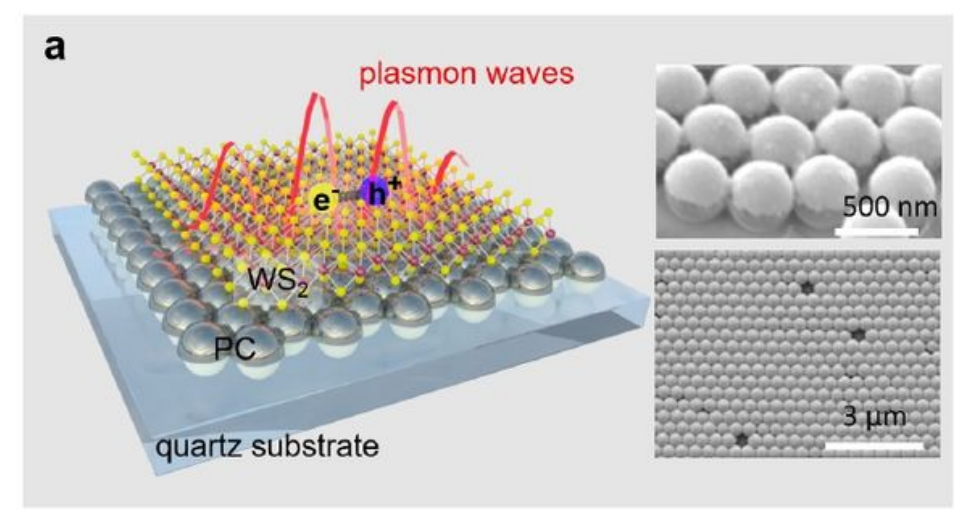

b

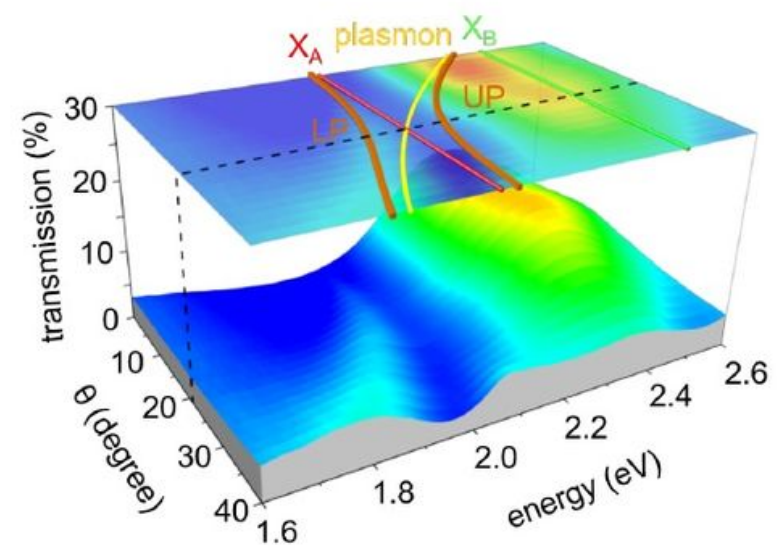

Figure 1

Structures of a PC-WS2 sample and steady-state optical properties. a, schematic of polariton formation in a WS2 ML that is supported on a self-assembled plasmonic crystal. The Al2O3 spacer is not depicted for similicity. right insets: side and top-view scanning electron microscope (SEM) images; $b$, angle-resolved transmission spectra under $p$-polarised illumination and their projection (top $x-y$ plane), in which the spectral positions of exciton $A(X A)$ and $B(X B)$, calculated dispersions of plasmon lattice modes (yellow curve), and upper and lower branches of polaritons (orange curves) are indicated. The tuned angle $(0=$ 220) is marked with a blacked dahsed line. Refer to Section 1 in the SI for detailed discussion of the strong plasmon-exciton coupling and its dispersion. 

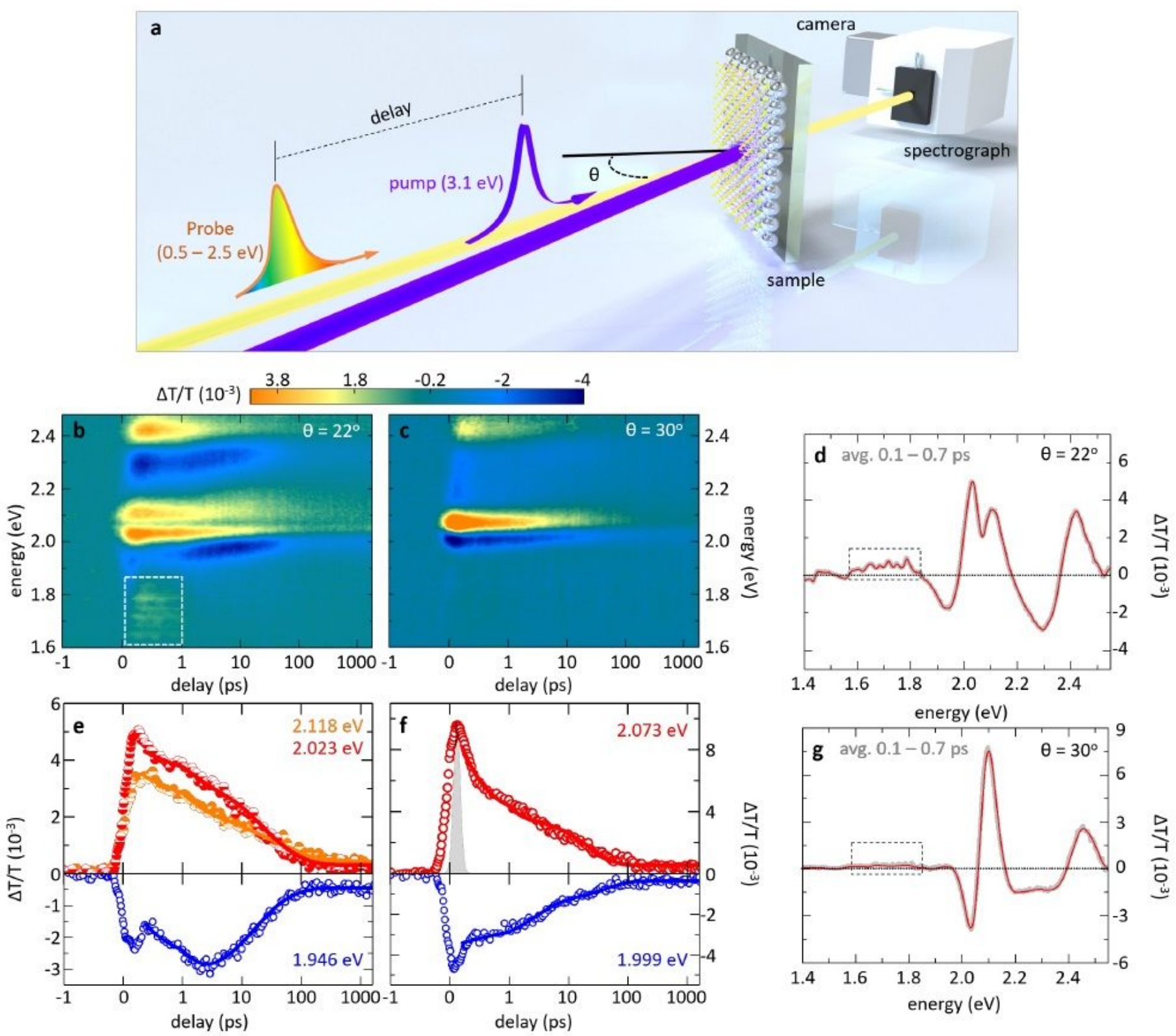

\section{Figure 2}

Transient optical responses of PC-WS2 systems. a, schematic of angle-resolved ultrafast pumpprobe spectroscopy; (note: the pump and probe beams are nearly colinear in practical experiments) $b, d$ and e refer to normalised differential transmission spectra $(T=T)$ at the tuned angle $(0=220)$, while $c$, $f$ and $g$ refer to $T=T$ at the detuned angle $(0=300) ; b$ and $c$ are intensity plots of $T=T$ as function of time delay and probe photon energy, using the same colour bar (which is also used by Fig.3a); $d$ and $g$ are $\mathrm{T}=\mathrm{T}$ spectra averaged within the time span from 0.1 to $0.7 \mathrm{ps}$ after pump; e and $\mathrm{f}$ are $\mathrm{T}=\mathrm{T}$ transient at specific energies (labelled with different colours), in which scatter symbols and solid curves represent measured and fitted data, respectively. Dashed frames in panel $b, d$ and g mark the spectral region of the broad maxima (see main text). All measurements were carried out using $400 \mathrm{~nm}(E=3: 1 \mathrm{eV})$ pump pulses that have $100 \mathrm{fs}$ duration and pump fluence of $12 \mathrm{uJ} / \mathrm{cm} 2$ at room temperature. The instrumentresponse-function is shown as the grey area in panel f. All time axes are displayed in logarithmic scales. 
a

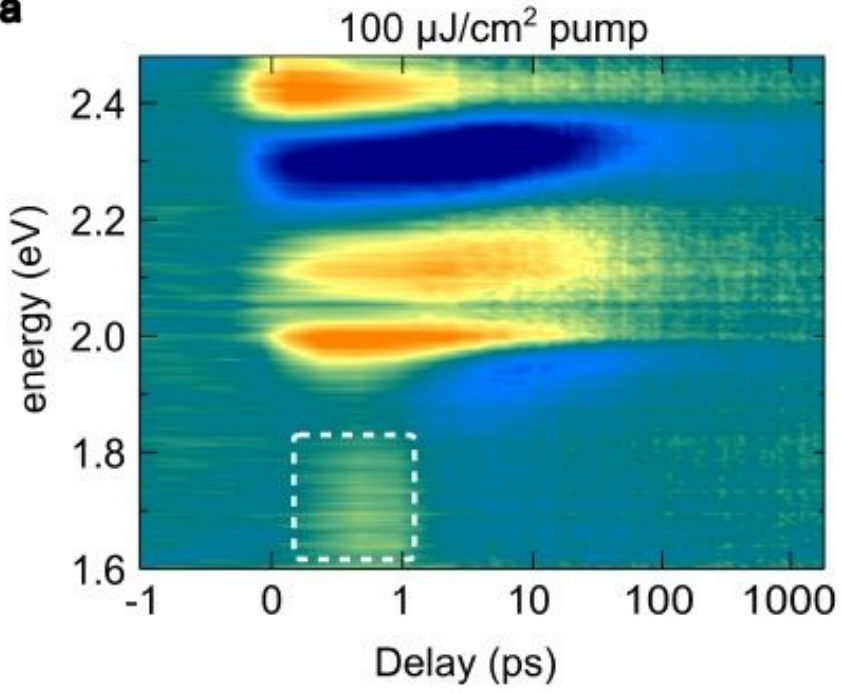

b
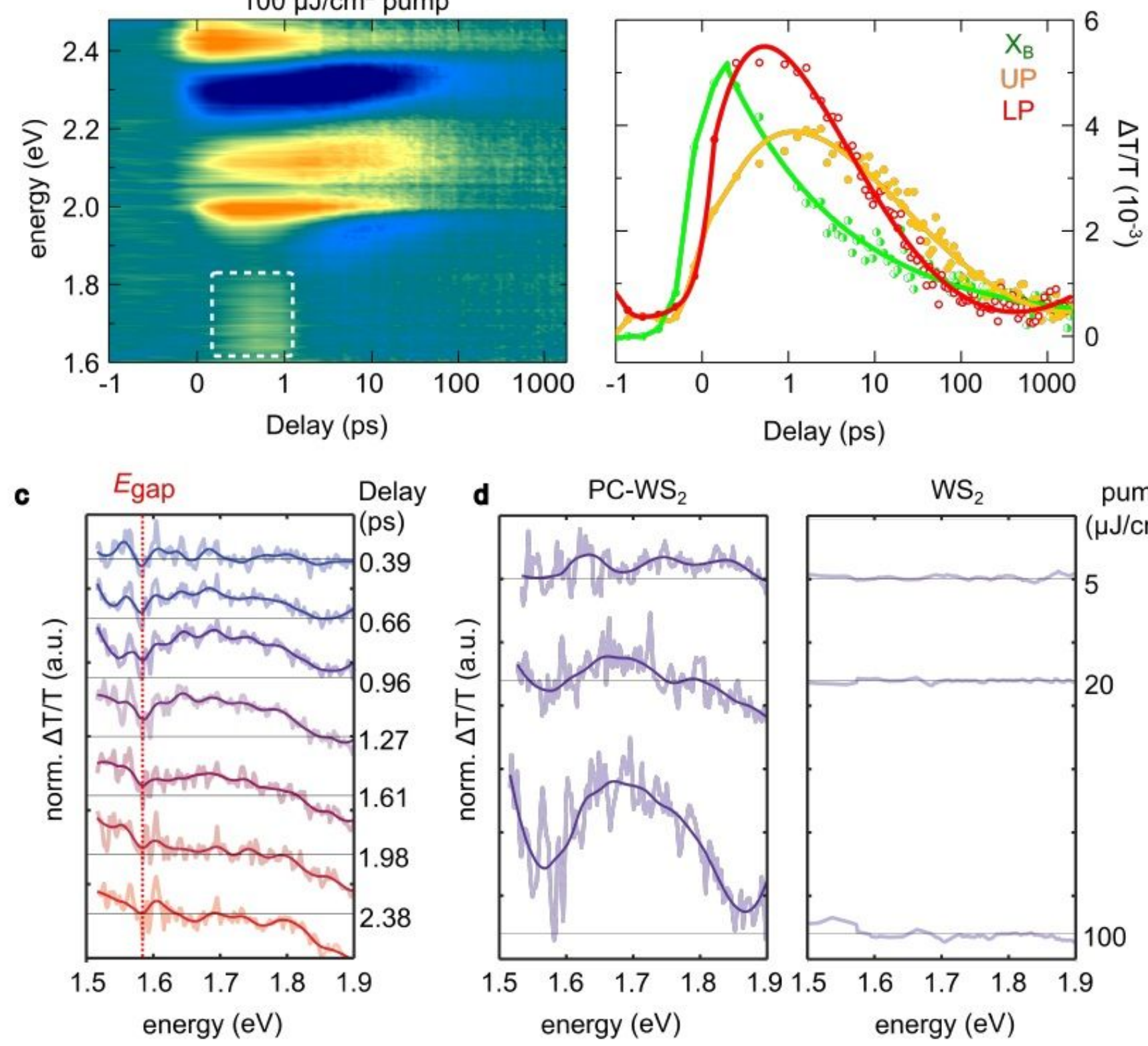
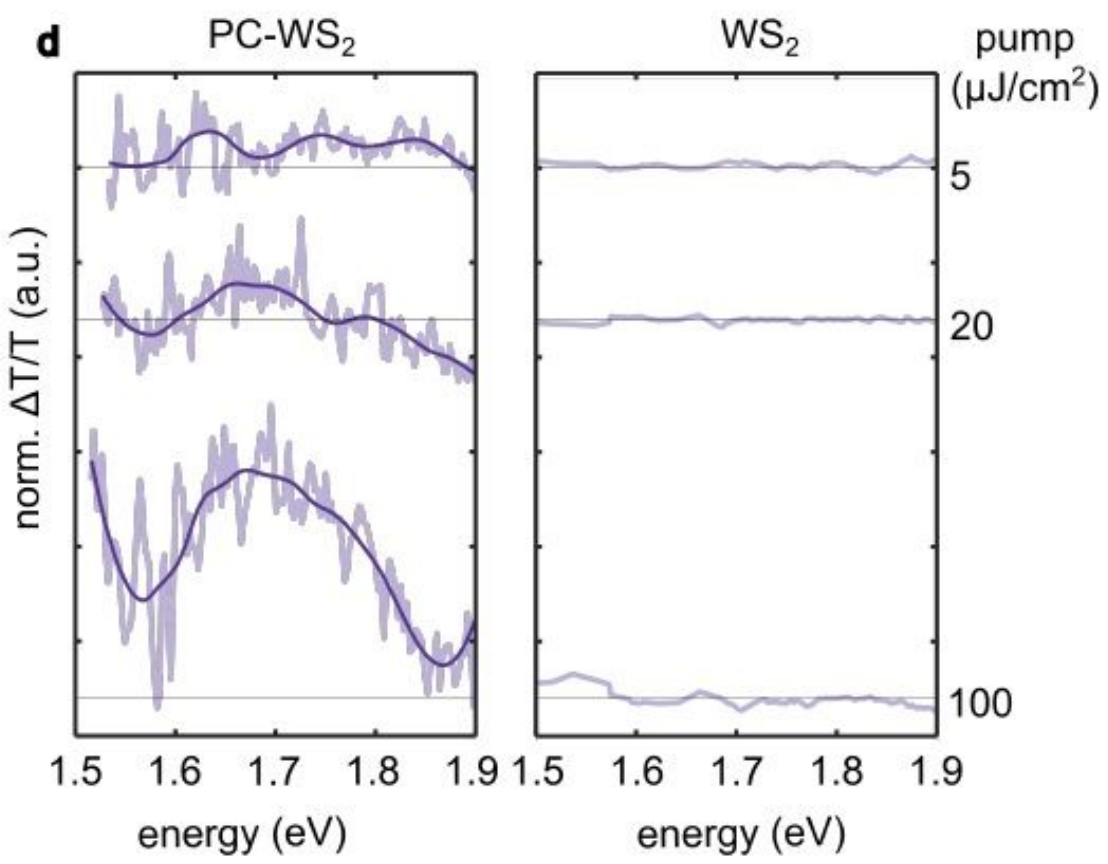

Figure 3

Transient optical responses under high-power pump. a, intensity plot of $\mathrm{T}=\mathrm{T}$ spectra of PCWS2 under $100 \mathrm{uJ}=\mathrm{cm} 2$ pump fluence at $0=22 \mathrm{o}$, where orange (blue) colour represents the maximum (minimum) value. $b$, delay time dependent spectra $(T=T)$ at energies of UP, LP and exciton B extracted from panel a. Solid curves are plotted only for visual guidance $c, T=T$ spectra at different delay times, extracted from the white dashed frame in panel a; red dashed vertical line indicates the onset of renormalised bandgap. $\mathrm{d}$, comparison of T=T spectra at delay of 0.96 ps between PC-WS2 (left) and WS2 MLs (right) under gradually increasing pump fluence. 


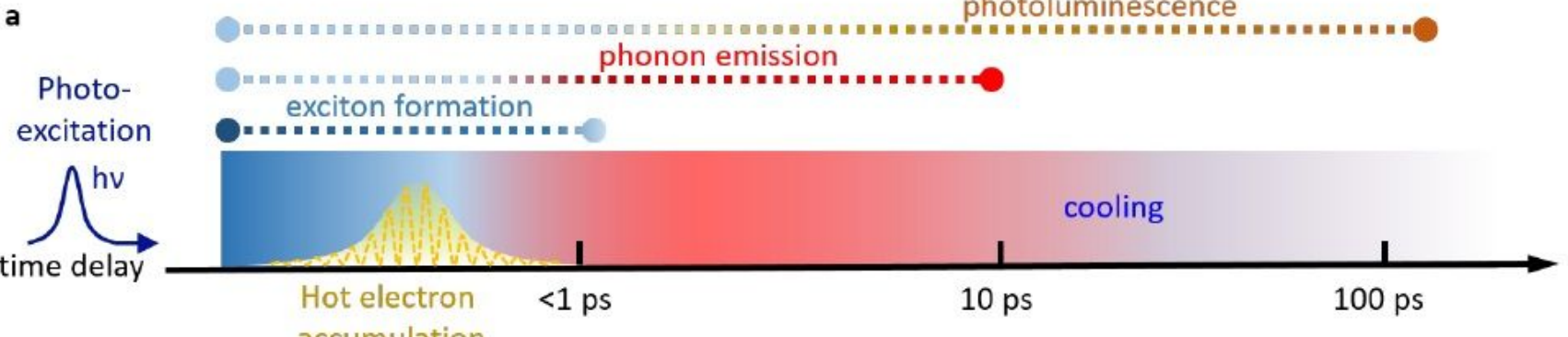

accumulation
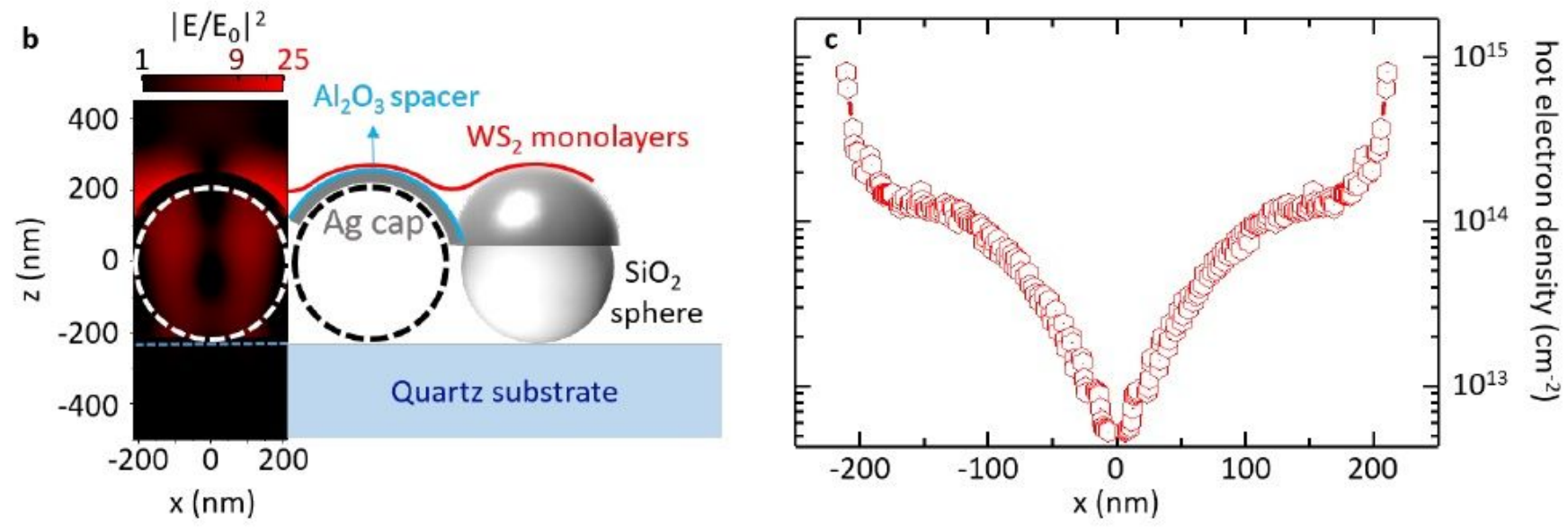

Figure 4

Hot electron density and distribution. a, schematic illustration of the overall relaxation scenario after pulsed optical excitation on the PC-WS2 systems, where the yellow oscillating dashed curve stands for repeated hot electron population in lattice; $b$, simulated intensity enhancement $j E=E 0 j 2$ in a single spherecap unit (XZ cross-section at middle of the sphere) at the frequency of $2.05 \mathrm{eV}$, together with a schematic of the PC-WS2 sample; please note that the WS2 ML is partially suspended at interstices between two Ag caps, but not fully covering the metal surface; detailed morphology can be referred to our previous work[15]; c, calculated hot electron density along the curved WS2 surface as a function of projected distance along $\mathrm{x}$-direction (at the same cross-section as in panel b).

\section{Supplementary Files}

This is a list of supplementary files associated with this preprint. Click to download.

- SIDing.pdf 